\title{
Fundamentality and Conditionality of Existence
}

Sahana Rajan*

\begin{abstract}
In metaphysics, fundamentality is a central theme involving debates on the nature of existents, as wholes. These debates are largely object-oriented in their standpoint and engage with composites or wholes through the mereological notion of compositionality. The ontological significance of the parts overrides that of wholes since the existence and identity of the latter are dependent on that of the former. Broadly, the candidates for fundamental entities are considered to be elementary particles of modern physics (since they appear to play the role of ultimate parts to all phenomena). The paper intends to show the inadequacy of the object-oriented notion of conditionality by pointing out that the parts and wholes possess varying conditions of existence. By alleging that only the parts are ontologically significant is to conflate such conditions and neglect the spectrum of conditions which exist in our world. A proposal for a revised notion of compositionality in terms of structural relatedness is also put forward.
\end{abstract}

Keywords: Compositionality, Object-Oriented Metaphysics, Conditionality of Existence

\section{Introduction}

In contemporary metaphysics, one of the significant themes is that of fundamentalityi. The notion of fundamentality attempts to

* University of Delhi, India; sahana.rajan18@gmail.com 
formulate that which is primitive or basic about our reality. The predominant characterisation of fundamentality is in terms of ontological independence. It can be written as:

Principle of Ontological Independence: $x$ is fundamental if and only if there is no $y$ such that $x$ is dependent on it for its existence and identity.

The above formulation can take different forms: absolute, relative and restricted fundamentality. Barring the few contemporary theories of fundamentality which preserve composites as significant inhabitants of their ontology, the prevalent understanding of the three forms of fundamentality posit ultimate independent entities from which the composites are derived. In a sense, the ontological significance of an entity is directly proportional to the extent of its ontological independence.

The broad claim of fundamentality is that the explanation of that which is fundamental about our world would render a complete metaphysical picture of the world. Fundamentality usually takes the form of fundamental entities or a fundamental level of reality. Entities which are fundamental or which constitute the fundamental level of reality are ontologically independent and compose all other entities. This is the standpoint of object-oriented realism in contemporary metaphysics (Thalos, 2011).

\section{Object-Oriented Notion of Compositionality}

The relation between such fundamental entities and the composites is based on the notion of compositionality which treats wholes or composites as mereological sums of their parts.

In classic extensional mereology, mereological sums or fusions or wholes are unstructured entities. Analogous to the axiom of extensionality in set theory, "the existence and identity of a mereological sum is determined exclusively on the basis of existence and identity of its parts; the arrangement or configuration of these parts is immaterial to the existence and identity of the sum they compose." (Koslicki, 2017). In this sense, composites or wholes are ultimately ontologically subservient to the fundamental entities which compose them. Such subservience is reflected in the 
treatment of composite entities simply as launching boards for discussions about fundamental entities.

\section{Fundamentality: In Physics and in Philosophy}

The representative of fundamental entities is generally considered to be elementary (subatomic) particles of contemporary physics, including quarks and leptons. However, this presupposes that the fundamentality in physics and in philosophy are similarly based. In physics, fundamentality is used interchangeably with elementary and refers to entities which do not have a substructure, that is, indivisible entities (Holden, 2004, p. 11; McGinn, 2011, p. 53). In philosophy, as mentioned above, fundamentality is defined in terms of ontological independence. Currently, this difference between the meaning of fundamentality in physics and philosophy has not been examined rigorously and there are no clear accounts of the relation between indivisibility and ontological independence.

\section{Conditionality of Existence}

One could conjecture that possible motivation for indivisible entities to be considered ontologically independent (and fundamental) is that the former does not have a structure or parts on which their identity or existence depends. However, such a motivation neglects the environmental conditions which elementary particles require to exist (Harrison, 2000, p. 427; Nave, 2018; Kolb, 2018, p. 70).

\section{Structural and Relational Conditions of Existence}

A distinction between two kinds of conditions for the existence of an entity is made to provide more clarity: structural and relational. Structural conditions of existence are those ways that the components of an entity must be arranged in, in order to enable its origin and sustenance. Relational conditions of existence are those ways in which the environment enables its origin and sustenance. The fulfilment of structural conditions would enable the formation and sustenance of internal structure while the fulfilment of relational conditions would enable obtaining of relational 
properties. Relational properties are those capacities that an entity possesses in order to respond to the changes in its environment. The internal structure enables and conditions the relational properties and vice-versa. Owing to limited space, this paper refrain from discussing this relationship in detail. It will suffice to point out that there are conditions for the existence of an entity and obtaining of such conditions enables the origin and development of its internal structure and its relational properties.

\section{Atoms, Us, the Earth, Universe and More}

There is overwhelming evidence to point to relational conditions of existence. This includes conditions for existence of atoms (Big Bang Nucleosynthesis (Petter, 2013, p. 76), conditions for existence of living organisations on Earth (molecular or chemical evolution of life (Davies, 2006), conditions for existence of communities (Turner \& Maryanski, 2015; Knight, 2018; Runciman, 2001), conditions for existence of Earth (Smart, 2015; Schmidt \& Lebedinsky, 2001), conditions for existence of Universe (Barrow, 2014) and so on. A more intuitive way of grasping the relational conditions is by considering counterfactual instances where one can engage with cases where the conditions are not met: suppose the temperatures on Earth had been different from as they were post-Big Bang and its effect on origin of atoms, if human beings did not possess the capacity to cooperate or inhibit their actions and its effect on origin of social groups, if an impact event had altered the way in which Solar System has developed and its effect on origin of Earth as a planet, if Big Bang had not happened.

\section{Violation of Principle of Ontological Independence}

Elementary particles do not have structural conditions for existence. However, they do have relational conditions for existence. If that is the case, then the Principle of Ontological Independence is violated. At this point, there are two options: either we accept that there are conditions of existence or that there are no conditions of existence. If there are conditions of existence and given that an ontologically independent being has no such conditions, we could move forward holding on to the idea of an 
impossible existent and engage with it; to be fair, God does fulfil the criterion of ontologically independent being. If there are conditions of existence, then there can be proposals for a criterion of existence which would be inclusive of such conditionality. Consider the following principle:

Principle of Conditionality of Existence $(\mathrm{PCE})_{\mathrm{Df}} \rightarrow$ To be is such that its possibility develops in obtaining of structural and relational conditions.

Given such conditionality of existence, we can build a notion of compositionality that does justice to both the structural and relational conditions of existence.

\section{Wholes as Environments for Parts}

Before formulating the notion, I would like to note a significant point regarding the existence of quarks and other subatomic particles in our world today. The only conditions under which the quarks exist today is as parts of entities which have responded effectively to the changing environment. From the air which is composed of oxygen, nitrogen, carbon dioxide and argon molecules to the organisms composed of functional organ systems, quarks and other subatomic particles exist as constituents to composites. If anything, that we are all made of quarks and other elementary particles is a lesson in ontological dependence! Diversity in the kinds of organisations we encounter are attempts at responding to the environment, under changing set of conditions.

It should be observed that the ontological consequence of claiming that the existence and identity of a whole depend on that of its parts. Given that the parts and the whole have varying conditions of existence, such a claim conflates these conditions. The preexistent mereological notion of compositionality fails to differentiate between a spectrum of environmental conditions. In the following section, this paper proposes a revised notion of compositionality, in terms of structural relatedness. 


\section{Compositionality in terms of Structural Relatedness}

Consider the following notion of compositionality:

Compositionality in terms of Structural Relatedness $\left(\mathrm{C}_{\mathrm{SR}}\right)$ : An existent $x$ is a structure $S$ composed of components $\left[\mathrm{C}_{1}\right.$, $\left.C_{2}, C_{3} \ldots C_{n}\right]: S\left\{C_{1}, C_{2}, C_{3} \ldots C_{n}\right\}$ such that $x$ forms the environment (a set of conditions) within which the components can sustain their internal structure and relational properties.

\section{Structures and Coalescence}

The metaphysical picture of the world drawn with the revised notion of compositionality, along with the Principle of Conditionality of Existence is: The actual world is a continuous developing of existents, grasped as structures. There is a diverse variety of existents; such existents can be classified into different kinds. The existents have structural and relational conditions of existence. The obtaining of such conditions enables their origin and development. The set of conditions in which an existent is embedded is considered to be its environment and it forms the horizon of its sustenance. As environmental conditions change, the existents respondii. Such a response involves the sustenance of its internal structure.

Coalescence is the ontological merging of an existent with other existents belonging to the same kind. Under such coalescence, the existent preserves its internal structure. However, the merge with the other existents alters its relational properties. It is the nature of coalescence that determines the furtherance or constraint on the relational properties of involved existents. Different kinds of wholes can be formed through such coalescence. Such wholes are characterised by their individual internal structure, nonaggregative to structures of the existents which constitute it (Wimsatt, 2000). This is due to the relational efficacy of the coalescence. As environmental conditions change, such wholes can themselves become constituents to other wholes. In this way, reality consists of such continuous developing of a diverse range of wholes, interacting with the environment- which are sustaining 
their internal structures and negotiating their relational properties through coalescence.

The space for studying fundamentality through structural relatedness in contemporary metaphysics can be built through the justification of the ontological significance of different kinds of wholes. This can be done by understanding fundamentality as specific to levels of reality ${ }^{3}$ (De Haro, 2018).

\section{Conclusion}

The inclusion of conditionality of existence in the notion of fundamentality will provide a precise answer to the question of whether supposed ontologically independent entities can exhaust the metaphysical picture of our world. It will be a negative answer and the concept of compositionality in terms of structural relatedness is shown to be providing a fertile ground for justifying this inclusion.

\section{References}

Barrow, J. D. (2014). The origin of the universe. Orion. Retrieved from https:/ / books.google.co.in/books?id=KHPhAgAAQBAJ

Davies, P. (2006). The origin of life. Penguin Books Limited. Retrieved from https://books.google.co.in/books?id=JQt2i8HKN4C

De Haro, S. (2018). Relative fundamentality and the metaphysics of emergence. Retrieved from https://fqxi.org/data/essaycontest-files/De_Haro_180101_Fundamentali.pdf

Harrison, E. (2000). Cosmology: The science of the universe. Cambridge University Press. Retrieved from https:// books.google.co.in/ books?id= eFchAw AAQBAJ

Holden, T. (2004). The architecture of matter: Galileo to kant. Oxford University Press.

Knight, C. (2018). Origins of society. Retrieved from http://radicalanthropologygroup.org/sites/default/files/pdf/ pub_origins_hs.pdf

Kolb, E. (2018). The early universe. CRC Press. Retrieved from https://books.google.co.in/books?id=FwpQDwAAQBAJ

Koslicki, K. (2017). Structure. In Handbook of mereology. 
McGinn, C. (2011). Basic structures of reality: Essays in meta-physics. Oxford University Press.

Nave, C. R. (2018). Elementary particle populations. Georgia State University. Retrieved from http://hyperphysics.phyastr.gsu.edu/hbase/Particles/parpop.html

Petter, P. (2013). Basic knowledge of Astrophysics: A new way. epubli $\mathrm{GmbH}$. Retrieved from https://books.google.co.in/ books? id=Ne69AQAAQBAJ

Runciman, W. G. (2001). The origin of human social institutions. British Academy.

Schmidt, O., \& Lebedinsky, A. (2001). A theory of earth's origin: Four lectures. University Press of the Pacific. Retrieved from https://books.google.co.in/books?id=E1Ast8BJQI8C

Smart, W. M. (2015). The origin of the earth. Cambridge University Press. Retrieved from https:// books.google.co.in/ books? id=0BVEBgAAQBAJ

Tahko, T. E. (2018). Fundamentality. In E. N. Zalta (Ed.), The Stanford encyclopedia of philosophy (Fall 2018). Metaphysics Research Lab, Stanford University.

Thalos, M. (2011). Two conceptions of fundamentality. Philosophy of the Social Sciences,41(2), 151-177.

Turner, J. H., \& Maryanski, A. (2015). On the origin of societies by natural selection. Taylor \& Francis. Retrieved from https:/ / books.google.co.in/books?id=C2TvCgAAQBAJ

Wimsatt, W. C. (2000). Emergence as non-aggregativity and the biases of reductionisms. Foundations of Science, 5(3), 269-297.

\section{Endnotes}

i Refer to (Tahko, 2018) for an overview of concept of fundamentality.

ii It is important to note that response does not presuppose agency. Consider the case of water turning into vapor: Suppose water is heated slowly in a container. The molecules in the water begin to move. Some of these molecules move faster than the others. After a point, the molecules move fast enough to break the hydrogen bonds which generally holds the water molecules together. This releases the individual molecules into the air. This process of evaporation is affected by many factors including humidity, air pressure and 
temperature. In this example, there is no agency. Response is a series of changes which an existent realises in alignment with the difference in environment. Different kinds of wholes have varying response to environmental changes. Certain kinds of wholes also include the capacity to regulate their internal structure as a response. 